

\title{
Gestión de la Diversidad Cultural: recursos y herramientas del Trabajo Social
} Management of Cultural Diversity: Resources and Tools of Social Work

\author{
Esther Raya-Diez*, Maria Ezquerro Sáenz*, Cecilia Serranno-Martinez ${ }^{* *}$ \\ * Universidad de La Rioja. esther.raya@unirioja.es \\ *** Entretanto Entretente-Bitartean Jolasean. marezsa@hotmail.com \\ *** Universidad de La Rioja. cecilia.serrano@unirioja.es
}

\begin{abstract}
:
We live in social and cultural diverse societies. UNESCO has pointed out the richness of cultural diversity in economic and social development. However, the confluence in the territory of people with different culture, customs, language and / or religions is often a source of conflict. There are different situations of sociability that lead to different ways of managing cultural diversity with different results in the axis of exclusion inclusion. The article analyses the management of cultural diversity as an area of Social Work intervention. Also, it presents professional competences, available resources and examples of diversity management activities in different areas of intervention.
\end{abstract}

Keywords: Interculturality; methodology; social intervention; migration; community work; inclusion.

\section{Resumen:}

Vivimos en sociedades diversas social y culturalmente. La Unesco ha señalado la riqueza de la diversidad cultural en el desarrollo económico y social. Sin embargo, la confluencia en el territorio de personas con diferente cultura, costumbres, lengua y/o religiones suele ser fuente de conflicto. Existen diferentes situaciones de sociabilidad que conllevan a diferentes formas de gestionar la diversidad cultural con resultados distintos en el eje inclusión exclusión. En el artículo se analiza la gestión de la diversidad cultural como ámbito de intervención del Trabajo Social. Se presentan las competencias profesionales, los recursos disponibles y se muestran ejemplos de actividades de gestión de la diversidad en diferentes ámbitos de intervención.

Palabras clave: Interculturalidad; metodología; intervención social; migración; trabajo comunitario; inclusión.

\section{Article info:}

Received: 14/02/2019 / Received in revised form: 05/07/2019

Accepted: 08/07/2019 / Published online: 29/07/2019

DOI: http://dx.doi.org/10.5944/comunitania.18.4 


\section{Introducción}

El concepto de cultura ha sido ampliamente estudiado por las ciencias sociales. Desde la antropología y la sociología se han aportado definiciones para el análisis de la misma, con autores como Tylor, Kroeber, Boas, Malinowski, White, Huxley, entre otros. Tomamos como punto de partida la definición clásica que define la cultura como "Sistema de creencias, valores, costumbres, conductas y artefactos compartidos, que los miembros de una sociedad usan en interacción entre ellos mismos y con su mundo, y que son transmitidos de generación en generación a través del aprendizaje" (Plog y Bates 1980). La cultura tiene implicaciones en la satisfacción de las necesidades (Löwy y Gundel 2006). La cultura es un aprendizaje, y por tanto dependiente del contexto en el que se vive y de las oportunidades que el mismo ofrece a las personas. Da sentido a la realidad, permite interpretarla a partir de las claves aprendidas. Es un todo integrado (creencias, valores, costumbres, conductas, artefactos) que la persona que la ha adquirido la comprende sin cuestionamientos. Se comparte diferencialmente y es un dispositivo de adaptación.

Estos preceptos culturales se ven modificados en un contexto de diversidad cultural. Cada vez es más difícil encontrar sociedades o comunidades monoculturales. Si la historia de la humanidad puede ser analizada en clave de contactos multiculturales, en la actualidad, la globalización llega a todos los rincones del planeta y con ello una suerte de diversidad cultural, fuertemente acelerada a través de las nuevas tecnologías. Además de la interacción digital entre personas de diferentes países y culturas, los movimientos migratorios implican el establecimiento de nuevos tipos de relación, originados por la fusión de culturas diferentes en un mismo territorio.

Según Giménez, Álamo y Pérez del Olmo (2015) hay tres situaciones de sociabilidad que podemos encontrar en contextos de diversidad cultural, que implican una manera u otra de relacionarse en el territorio. Estas situaciones son: hostilidad, coexistencia y convivencia.

En primer lugar, la hostilidad se sitúa en el extremo negativo, en tanto que sí que existe relación, pero ésta no es buena. Se tratan de relaciones interpersonales caracterizadas por la desconfianza, el recelo y otros tipos de violencia que pueden variar desde la física hasta la simbólica y que pueden manifestarse de manera clara y directa o bien latente. En un contexto en el que existe la hostilidad se pueden dar situaciones de odio y rechazo a otras culturas, que se pueden manifestar desde la culpabilización de los males ocasionados en el entorno inmediato hacía aquellos con diferente cultura u origen, hasta otras expresiones de rechazo en lugares concretos del espacio (lugares de culto, mercados, etc.).

En segundo lugar, la coexistencia se sitúa en un término medio e implica que el 
conjunto de personas que integran la comunidad, cohabitan en ese espacio y tiempo. No obstante, la característica principal de la coexistencia es que no hay relación y se dan situaciones estancadas y pasivas. En este sentido, pueden observarse realidades sin problemas patentes, pero con unas relaciones caracterizadas por la fragilidad y la inexistencia de relaciones, ni en sentido positivo (convivencia) ni en sentido negativo (insultos, agresiones, etc.). Por ello la coexistencia es una manera estática de estar en un mismo espacio.

En tercer lugar, la convivencia se sitúa en el extremo positivo ya que implica relaciones pacíficas caracterizadas por el respeto y el diálogo. Esto no significa que no existan conflictos, si no que la manera de relacionarse y enfrentarse a los problemas es por medio de la palabra y utilizando estrategias alternativas de resolución de conflictos, como la mediación y la negociación, entre otras. Se tratan de relaciones carentes de violencia que implican una exigencia en el mantenimiento de las mismas por parte de los integrantes en este tipo de comunidades, espacios o situaciones caracterizadas por la confluencia de culturas.

Como se puede extraer de lo anterior, el ideal último a alcanzar es el de una comunidad caracterizada por la convivencia. Si bien esto es una cuestión de gestión de las políticas públicas, donde el papel delTrabajo Social resulta relevante.

En el presente artículo nos ocupamos de esta cuestión, a través de cuatro apartados. En el primero se hace una referencia conceptual a la diversidad cultural y su gestión; en el segundo, se presentan las competencias en cuanto conocimientos, habilidades y destrezas del profesional para la gestión de la diversidad cultural; en el tercer apartado se presentan una serie de recursos sobre el tema y finalmente en el cuarto apartado se presentan ejemplos de actividades concretas desarrolladas para abordar la diversidad cultural en diferentes contextos.

\section{Diversidad cultural y su gestión}

Desde la antropología cultural se han estudiado las actitudes que pueden adoptarse ante la existencia de otras culturas. En el siguiente cuadro se presentan de forma resumida las diferencias entre etnocentrismo, relativismo cultural e interculturalidad: 


\section{CUADRO 1: Actitudes ante la existencia de otras culturas}

\begin{tabular}{|l|l|l|}
\hline \multicolumn{2}{|c|}{ Actitud tipo } & \multicolumn{1}{c|}{ Definición } \\
\hline \multirow{2}{*}{ Etnocentrismo } & Guetización & $\begin{array}{l}\text { Considerar la supremacía de la propia cultura } \\
\text { frente a las demás }\end{array}$ \\
\hline \multirow{2}{*}{$\begin{array}{l}\text { Relativismo } \\
\text { cultural }\end{array}$} & $\begin{array}{l}\text { Separación de las minorías en espacios } \\
\text { específicos. No hay contacto entre la cultura } \\
\text { mayoritaria y la minoritaria, aunque se } \\
\text { respeten sus costumbres. }\end{array}$ \\
\cline { 2 - 3 } & Falta de sentido crítico & $\begin{array}{l}\text { Etnocentrismo inverso. Deslumbrado por los } \\
\text { aspectos positivos de la otra cultura, } \\
\text { establece su defensa por encima de la crítica. }\end{array}$ \\
\cline { 2 - 3 } & Conservacionismo & $\begin{array}{l}\text { Mantiene una visión estática de las culturas y } \\
\text { un ahistoricismo; recomienda conservar las } \\
\text { culturas tradicionales. }\end{array}$ \\
\hline \multirow{2}{*}{ Interculturalismo } & $\begin{array}{l}\text { Compartir y aprender de las culturas con el } \\
\text { fin de promover el entendimiento. Se } \\
\text { promueve el respeto a otras culturas a la vez } \\
\text { que se desarrolla una actividad crítica. }\end{array}$ \\
\hline
\end{tabular}

Fuente: Elaboración propia a partir de la revisión bibliográfica.

Cada una de ellas comporta formas diferentes de interacción en el territorio que puede generar problemas de convivencia, cuando no de discriminación y xenofobia. De ahí la necesidad de gestionar la diversidad cultural. Concretamente, en el acercamiento entre culturas se dan cuatro situaciones prototípicas, en función de la relación entre la conservación de la identidad cultural y sus costumbres y la valoración de las relaciones entre las diferentes culturas, como puede verse en el siguiente cuadro:

\section{CuADro 2: Acercamiento entre Culturas}

\begin{tabular}{|c|c|c|c|}
\hline & & \multicolumn{2}{|c|}{$\begin{array}{l}\text { Se conserva la identidad cultural y las } \\
\text { costumbres }\end{array}$} \\
\hline & & $\mathrm{Si}$ & No \\
\hline \multirow{2}{*}{$\begin{array}{c}\text { Se valoran las relaciones entre } \\
\text { las culturas }\end{array}$} & $\mathrm{Si}$ & INTEGRACIÓN & ASIMILACIÓN \\
\hline & No & SEPARACIÓN & MARGINACIÓN \\
\hline
\end{tabular}

Fuente: Elaboración propia a partir Martin (2003).

Cuando no se cumple ninguna de las dos condiciones se produce la marginación. En este caso no se facilita el mantenimiento de la diversidad, ni tampoco las relaciones intergrupales. Se busca la asimilación cultural y lingüística por medio 
de una estrategia de separación de aquellos que son diferentes, normalmente cuando se percibe la diferencia como la causa de la desigualdad, y, por lo tanto, se trata de eliminarla.

Por su parte, la asimilación supone una uniformización cultural. Las minorías culturales van adoptando la lengua, los valores, las normas y señas de identidad de la cultura dominante, y al mismo tiempo perdiendo su identidad y cultura propia.

En una situación opuesta se encuentra la separación. En este caso se respetan las diferencias culturales manteniéndose la identidad, la cultura y la lengua de origen, pero no se promueve la interacción entre los grupos socioculturales diversos. Esto se puede traducir en segregación social y guetización espacial.

\section{GrÁfICo 1. Tipología de modelos sociopolíticos ante la diversidad sociocultural}

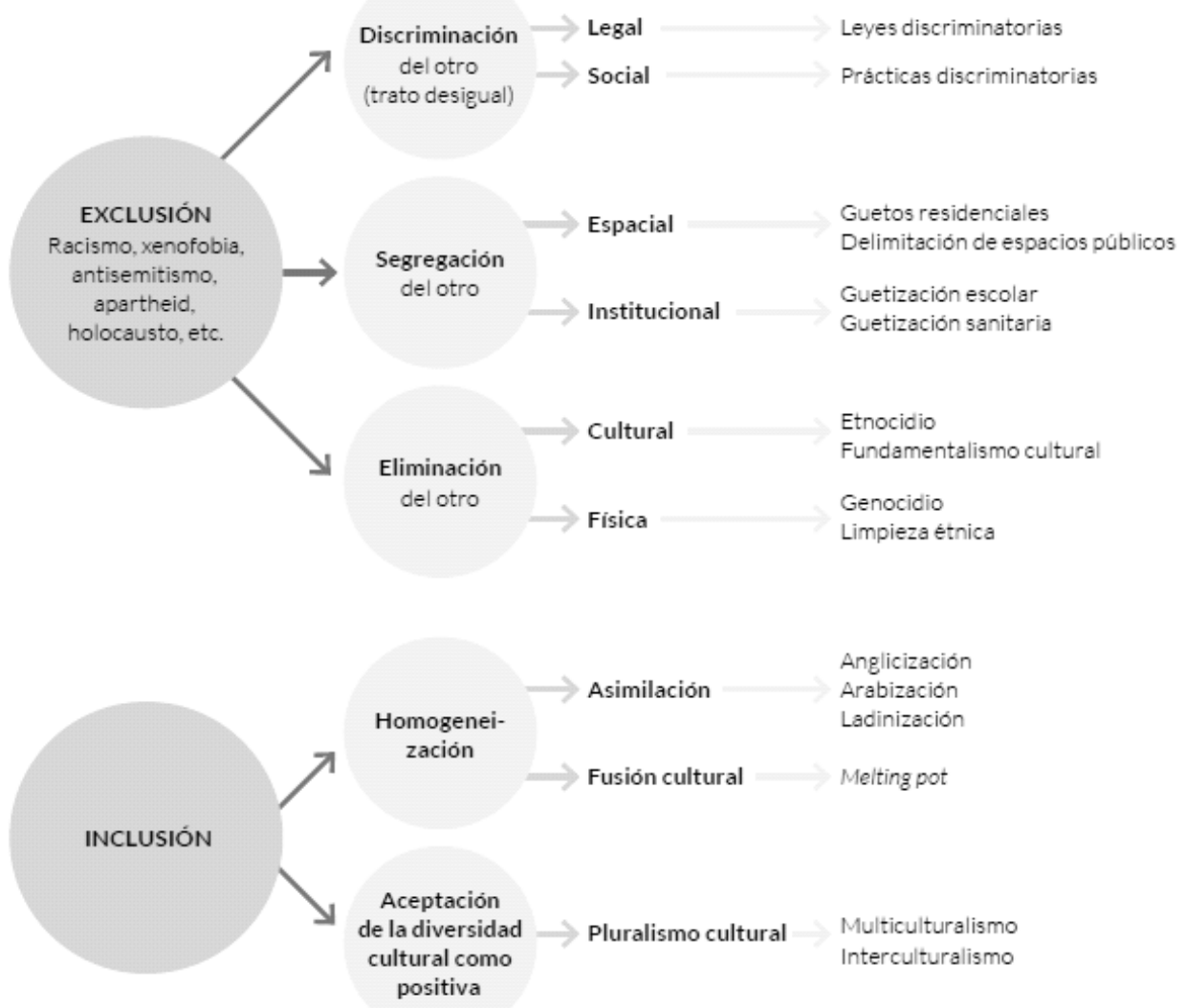

Fuente: Giménez, Álamo y Pérez del Olmo (2015:50). 
Finalmente, la integración sería el modelo de gestión de la diversidad que permite que un sujeto o grupo que se encuentra en un espacio marginal pase a participar de la dinámica social mayoritaria de forma normalizada. Permite la incorporación de una cultura e identidad nuevas, así como el mantenimiento de una identidad y cultura diferentes. Esto se consigue manteniendo la diversidad y al mismo tiempo fomentando las relaciones intergrupales.

Desde un punto de vista analítico se puede señalar que, en la tipología de modelos sociopolíticos ante la diversidad sociocultural aparecen la exclusión y la inclusión (Giménez, 2003; Giménez, Álamo y Pérez del Olmo 2015), cada uno de ellos generadores de diferentes formas de relación. Dentro de los modelos de exclusión se incluye la discriminación, la segregación, y la eliminación. En segundo lugar, la inclusión comprende la homogenización y la aceptación de la diversidad cultural como positiva.

Los modelos de gestión de la diversidad que se han ido aplicando en diferentes lugares, están relacionados con la comprensión que se tenga de la relación entre igualdad y diferencia entre personas y culturas. En los modelos de inclusión se encuentran la asimilación, la fusión cultural, el multiculturalismo y el interculturalismo. Para Giménez (2003) los dos primeros se agruparían en una categoría de "inclusión aparente" relacionándolos con la homogeneización cultural y los dos siguientes como modelos de "inclusión real" basada en la aceptación de la diversidad cultural como positiva.

Se habla de Multiculturalidad cuando grupos étnicos diferentes, con una lengua y una cultura diferentes, conviven en un mismo espacio geográfico y social. Se trata de cohabitación de las culturas, con escasa influencia. Por su parte el concepto de Interculturalidad se refiere a la situación en la que personas de diferentes culturas, conviven e interactúan, con intercambios culturales que pueden generar una cultura nueva. El interculturalismo basa sus principios en el respeto a las diferencias, énfasis en lo común, igualdad de derechos e interacción positiva. "Si en el movimiento multiculturalista el acento está puesto en cada cultura, en el planteamiento intercultural lo que preocupa es abordar la relación entre ellas". (Giménez: 2003,13). Por ello, para que tenga lugar la interculturalidad ésta ha de ir dirigida a superar la exclusión y superar los modelos de inclusión, promoviendo los vínculos y la relación entre culturas. La interculturalidad aboga por los derechos de las personas inmigrantes a crear, junto a las personas autóctonas, una nueva ciudadanía.

En la Declaración Universal de Derechos Humanos (1948) donde se reconoce los mismos derechos a todas las personas sin discriminación por razón de raza, nacionalidad o religión, se sientan las bases para la gestión de la diversidad cultural. La ONU a través de la UNESCO ha desarrollo diferentes instrumentos internacionales en favor de la diversidad cultural. En el siguiente cuadro se presenta el recorrido realizado en este sentido: 


\section{CUADRO 3: Instrumentos internacionales relacionados con la diversidad cultural}

\begin{tabular}{|c|l|}
\hline Año & \multicolumn{1}{c|}{ Instrumento internacional } \\
\hline 1950 & $\begin{array}{l}\text { Acuerdo sobre la Importación de Objetos de Carácter Educativo, Científico y } \\
\text { Cultural ("Acuerdo de Florencia") y su Protocolo de Nairobi de 1976 }\end{array}$ \\
\hline 1952 & Convención Universal sobre Derecho de Autor \\
\hline 1954 & $\begin{array}{l}\text { Convención sobre la Protección del Patrimonio Cultural en caso de Conflicto } \\
\text { Armado }\end{array}$ \\
\hline 1966 & Declaración de los Principios de la Cooperación Cultural Internacional \\
\hline 1970 & $\begin{array}{l}\text { Convención sobre las Medidas que Deben Adoptarse para Prohibir e Impedir la } \\
\text { Importación, la Exportación y la Transferencia de Propiedad Ilícitas de Bienes } \\
\text { Culturales }\end{array}$ \\
\hline 1972 & Convención sobre la Protección del Patrimonio Mundial, Cultural y Natural \\
\hline 1978 & Declaración sobre la Raza y los Prejuicios Raciales \\
\hline 1980 & Recomendación relativa a la Condición del Artista \\
\hline 1989 & Recomendación sobre la Salvaguardia de la Cultura Tradicional y Popular \\
\hline 2001 & Declaración Universal de la UNESCO sobre la Diversidad Cultural \\
\hline 2001 & Convención sobre la Protección del Patrimonio Cultural Subacuático \\
\hline 2003 & Convención para la Salvaguardia del Patrimonio Cultural Inmaterial \\
\hline 2003 & $\begin{array}{l}\text { Recommendation concerning the Promotion and Use of Multilingualism and } \\
\text { Universal Access to Cyberspace }\end{array}$ \\
\hline 2005 & $\begin{array}{l}\text { Convención sobre la Protección y la Promoción de la Diversidad de las } \\
\text { Expresiones Culturales }\end{array}$ \\
\hline 2011 & $\begin{array}{l}\text { Recomendación sobre el paisaje urbano histórico, con inclusión de un glosario de } \\
\text { definiciones }\end{array}$ \\
\hline 2015 & $\begin{array}{l}\text { Recomendación relativa a la protección y promoción de los museos y colecciones, } \\
\text { su diversidad y su función en la sociedad }\end{array}$ \\
\hline
\end{tabular}

Fuente: Página web de la Unesco www.unesco.org ${ }^{1}$.

En la Cumbre Mundial sobre el Desarrollo Sostenible, celebrada en Johannesburgo en septiembre de 2001, la Unesco presentó un documento titulado “Declaración Universal sobre la Diversidad Cultural" donde se reconocen las diferentes formas culturales y su valor como patrimonio de la humanidad. Se aboga por el desarrollo de políticas que promuevan el pluralismo cultural, "como respuesta política al hecho de la diversidad cultural" (Art. 2). Para avanzar en este sentido en el mismo documento se establecen una serie de líneas de acción y se presentan argumentos, principios y estrategias. Gestionar la diversidad cultural implica un proceso de trabajo consciente y decidido, a partir de una estrategia política a favor de la convivencia y la promoción de la diversidad de los grupos culturales existentes en el territorio.

Siguiendo a Rinken (2011) la gestión de la diversidad cultural se traduce en los siguientes aspectos: a) El respeto de todas las reglas y procedimientos que eviten un

1 http://www.unesco.org/new/es/culture/themes/normative-action/cultural-diversity/ 
trato discriminatorio; b) La voluntad y la capacidad para crear puentes y superar malentendidos, si surgieran; c) La disposición de conocimientos de los valores, comportamientos y actitudes existentes en distintas culturas, sobre todo los relacionados con las gestiones o actuaciones concretas: d) Un esfuerzo estratégico por consolidar el respaldo, por parte de la ciudadanía, en la igualdad de diferentes. Por su parte Portes y Vickstrom (2012) indican que la diversidad cultural, junto al incremento demográfico provocado por la llegada de inmigrantes, terminan por ser necesarios para alcanzar un relevo generacional que aporte energía y desacelere el bajo nivel demográfico; frente a la situación actual en la que las poblaciones se caracterizan por su edad avanzada y por ser homogéneas étnicamente. Por ello, esta diversidad no desafía la estructura de clases, sino la composición de las clases trabajadoras y supone "una división del trabajo compleja y basada en la solidaridad orgánica" (Portes y Vickstrom 2012:101). En suma, es necesario desarrollar estrategias de gestión de la diversidad cultural que posibiliten la generación de sociedades cohesionadas y fuertes.

Algunas de las estrategias para la gestión de la diversidad cultural pueden verse reforzadas por medio de un trabajo desde el ámbito educativo, sanitario, comunitario, etc. La educación intercultural ayudaría a procesar el fenómeno de la diferencia cultural y evitaría conflictos derivados de las diferencias culturales. Es más, por medio de este tipo de educación, "se ampliaría la curiosidad, el respeto y el interés por los diversos puntos de vista y daría la posibilidad de poner en entredicho los propios criterios culturales" (Escarbajal 2012:46). También en el ámbito de salud algunas de las acciones que se pueden desarrollar (Ramasco Gutiérrez y Garabato González 2015: 212-213), son:

- Mejorar los sistemas de información en salud, incluyendo variables que tengan en cuenta la diversidad sociocultural, tanto para tener información sobre la población inmigrante, como para aportar conocimiento en salud a la misma.

- Garantizar el acceso normalizado al sistema sanitario a la población inmigrante.

- Capacitar a los profesionales en salud y sensibilizarles en la gestión de la diversidad sociocultural.

- Mejorar la coordinación entre sectores y poner en común a las entidades y recursos que se dirigen a la población inmigrante.

Las estrategias desde el trabajo comunitario suponen la inclusión de espacios públicos como lugares de recreación identitaria, por los que se logra hacer que se conviertan en unos territorios "de encuentro, de reconocimiento, de intercambio de información y apoyo, así también como de ocio" (Martínez Aranda 2015:51). Estos espacios suponen para la población no autóctona un lugar para la recreación identitaria, identificación de simbolismos y la incorporación de estrategias de adaptación al nuevo contexto. 
Para poner en marcha este tipo de estrategias, uno de los desafíos de la acción social y de la búsqueda de alternativas desde el terreno científico es el de "combinar la inmediatez de la necesidad con la prevención" (Hernández Correa 2007:290) ante las diversas necesidades que surgen. Del mismo modo, no hay que olvidar las reflexiones de Marchioni, quien plantaba el reto de "salir del gueto de lo inmediato y saber trabajar de cara al futuro" (1989: 39).

Los profesionales vinculados a la gestión de la diversidad cultural, como son los y las trabajadoras sociales, tienen que dirigir las actuaciones a todos los colectivos por igual y no solo a las minorías incidiendo en los puntos comunes y buscando un acercamiento relacional entre las distintas culturas a través de una lógica de conocimiento y enriquecimiento mutuo.

Además de considerar los modelos de gestión de la diversidad cultural, hay que reconocer que los miembros de una determinada cultura en contacto con otras culturas pueden adoptar diferentes estrategias, que condicionarán necesariamente los mecanismos de desarrollo de los programas de gestión de la diversidad cultural. Las estrategias, en línea con la teoría de la disonancia cognitiva de Festinger (1957) pueden relacionarse con conductas adaptativas; defensivas o de ruptura, tal como se ha podido ver en las páginas anteriores. Todo lo planteado hasta el momento implica a los y las trabajadoras sociales la combinación de, al menos, tres elementos importantes que desarrollaremos en los siguientes epígrafes: competencias profesionales; recursos y actividades para trabajar la interculturalidad.

\section{Competencias profesionales}

La competencia profesional se refiere al conjunto de capacidades de diferente naturaleza (conocimientos, habilidades y actitudes) que permiten conseguir un resultado.

La competencia intercultural debe permitir a los profesionales que sean eficaces en contextos plurales. Como ha señalado Vázquez ser competente en la acción intercultural "no consiste únicamente en poder comunicarse con el otro diferente (...) sino captar los significados que se otorgan a los elementos que se comparten, teniendo en cuenta que comprender no implica ni aceptar, ni justificar ni descalificar automáticamente" (Vázquez 2002:130). Se trata de identificar las razones que explican el comportamiento diferente y para ello es necesario tener un conocimiento sobre el otro.

A partir de la propuesta de Vázquez aplicada alTrabajo Social, Essomba (2013) presenta de forma sintética el conjunto de conocimientos, destrezas y actitudes que implica la competencia intercultural de cualquier actor que se encuentra sumergido en una acción comunitaria intercultural, y por tanto aplicable a los agentes/ gestores culturales: 


\section{a. Conocimientos asociados a la competencia intercultural}

La importancia de la generación de confianza mutua en los procesos de ayuda.

La lógica de la comunicación.

El lugar de los inmigrantes en la estructura social.

La identidad y su proceso de configuración.

Las diferencias culturales y los ámbitos de conflicto.

Las influencias que distorsionan el conocimiento de la realidad.

Los proyectos migratorios.

Las implicaciones de la integración social de los inmigrantes.

Las propuestas de la interculturalidad.

Las habilidades para la acción intercultural.

La mediación y la resolución de conflictos.

b. Actitudes y emociones asociadas a la competencia intercultural

Valorar positivamente las aportaciones de los demás.

Apreciar la necesidad de establecer objetivos en común.

Dar importancia a la escucha y a la necesidad de entrenarse para el desarrollo de la comunicación.

Analizar y valorar críticamente las imágenes sociales dominantes respecto a la inmigración y los diferentes. Estereotipos.

Emitir mensajes positivos.

Empatía.

Desear ampliar nuestro conocimiento acerca del otro y de nosotros mismos.

Poder cuestionar comportamientos ajenos sin desvalorar a toda la persona.

Potenciar el respeto y la participación de todas las partes intervinientes en un conflicto.

c. Procedimientos asociados a la competencia intercultural

Dar y recibir confianza.

Establecer objetivos en común.

Manejar pautas que mejoran la comunicación.

Saber realizar e interpretar historias de vida de inmigrantes.

Manejar el uso de la comunicación no verbal en la relación de ayuda con inmigrantes.

Utilizar la descentración y la penetración en el sistema del otro como medio de ampliar nuestro conocimiento.

Ser capaz de transmitir mensajes positivos.

Saber analizar incidentes críticos.

Resolver conflictos fomentando la participación de las partes implicadas. 
Sirva esta clasificación como punto de partida para comprender las competencias profesionales inherentes a la diversidad cultural. Sin embargo, como señala Essomba (2013), no debe identificarse diversidad cultural únicamente con inmigración. Existe diversidad cultural también sin inmigración, como es el caso de la existencia de varias culturas autóctonas. En España el claro ejemplo es el de las cultura gitana y paya.

Asimismo, podemos hablar de una nueva figura bajo la que incluir acciones que promuevan relaciones e intercambios positivos dentro de contextos caracterizados por la diversidad cultural. El "mediador intercultural" se trata de un profesional con formación en interculturalidad y mediación, que desarrolla un trabajo de intermediación entre aquellos colectivos e instituciones que lo precisen, como, por ejemplo: entre población extranjera y autóctona, entre población migrante e instituciones, entre otros. Su labor fundamental es la de facilitar el diálogo ante situaciones conflictivas y ayuda a que las partes implicadas busquen por sí mismas la solución más adecuada. Para ello, ha de incorporar una serie de planteamientos metodológicos, que ayude a legitimar a todas las partes y trabajar la convivencia sin realizar diferencias por grupos culturales. En este sentido, los planteamientos que favorecerían una convivencia intercultural (Moreno 2006:30) son: a) Vías para la convivencia intercultural; b) Intervención en los contextos cotidianos; c) Trabajar con todos los grupos implicados.

En suma, gestionar la diversidad cultural implica partir de un interés por el otro y un reconocimiento a su valor como persona y como cultura. Para ello, es preciso saber gestionar recursos de diferente naturaleza, como se expone en el siguiente epígrafe.

\section{Recursos}

El término recurso es fuertemente polisémico. Entendemos por recurso todos los medios de los que dispone el profesional y la comunidad para articular su intervención en la realidad. Una definición clásica en Trabajo Social es la aportada por Patrocinio de las Heras y Elvira Cortajera que lo definen como los "medios humanos, materiales, técnicos, financieros, institucionales, etc. de que se dota a sí misma una sociedad para hacer frente a las necesidades de individuos, grupos y comunidades, en cuanto miembros integrantes de ella" (1985: 30). Por su parte Kisnerman (1984) diferenciaba los recursos según su naturaleza en a) recursos naturales (alimentos, energía); b) materiales (equipamiento, infraestructuras); c) técnicos (instrumentos, herramientas, procedimientos); d) financieros; e) humanos; f) institucionales. De todos ellos, nos interesa destacar la importancia de los recursos humanos, entendidos como las personas que participan en la relación, es decir, todos los actores presentes en el territorio, con sus conocimientos, capacidades, actitudes, etc. Es éste, el capital social sin duda el recurso más valioso de todo proceso de intervención en la 
realidad social. Una de las principales ideas a recordar sobre los recursos es que en los procesos de intervención en la realidad social el recurso más importante es el de las personas (ciudadanos, técnicos, políticos, etc.) presentes en el proceso, donde la creatividad y la comunicación son elementos clave. Moix nos recuerda en su definición del Trabajo Social, que es "la actividad de ayuda técnica y organizada, ejercida sobre las personas, los grupos y las comunidades, con el fin de procurar su más plena realización y mejor funcionamiento social, y su mayor bienestar, mediante la activación de los recursos internos y externos, principalmente los ofrecidos por los Servicios Sociales y por las instituciones y los sistemas del Bienestar Social" (Moix 2004:131). El papel de esta disciplina en la gestión de la diversidad cultural, consiste en gran medida en activar los recursos internos y externos para promover procesos de convivencia intercultural.

Para ello, es preciso que los y las trabajadoras sociales dispongan de información actualizada sobre diferentes recursos, los específicos del entorno y los compartidos, a través de las redes sociales, además de, promover los métodos y técnicas delTrabajo Social Comunitario para el desarrollo de comunidades inclusivas.

Centramos esta parte en presentar algunas guías a modo de recursos útiles para la gestión de la diversidad cultural. Es un listado que se presenta a modo de orientación que debe ser completado y perfeccionado por cada profesional en su ámbito de trabajo.

Además de los recursos, el conocimiento de buenas prácticas que se están desarrollando en torno a la gestión de la diversidad, puede servir de referencia para el desarrollo de la intervención aplicada al propio contexto de actuación o concibiendo nuevas a partir de su inspiración. A continuación, se describen algunos ejemplos de actividades que han sido desarrollados con éxito. 


\section{CUADRO 4: Recursos para la gestión de la diversidad cultural en diferentes ámbitos de actuación}

\begin{tabular}{|c|c|}
\hline \multicolumn{2}{|c|}{ Gestión de la diversidad cultural en el territorio: } \\
\hline $\begin{array}{l}\text { Hagamos de nuestro } \\
\text { barrio un lugar } \\
\text { habitable. Manual de } \\
\text { intervención } \\
\text { comunitaria en barrios. } \\
\text { Ceimigra }\end{array}$ & $\begin{array}{l}\text { Manual con un enfoque comunitario que aborda la convivencia intercultural y } \\
\text { aporta estrategias para fomentar la convivencia. Explica aspectos de la } \\
\text { convivencia ciudadana intercultural, así como la metodología de la intervención } \\
\text { comunitaria. Incluye la participación ciudadana como respuesta ante los } \\
\text { conflictos humanos y como herramienta para la implicación de todo el entramado } \\
\text { social.Se incluyen prácticas de Investigación-Acción Participativa. }\end{array}$ \\
\hline $\begin{array}{l}\text { Comprender la } \\
\text { diversidad, integración e } \\
\text { inclusión en } \\
\text { OPENCities }\end{array}$ & $\begin{array}{l}\text { Es la primera de las cuatro publicaciones del British Council que versan sobre el } \\
\text { Proyecto denominado: OPENCities (Ciudades Abiertas). Ofrece casos prácticos } \\
\text { de las ciudades participantes e instituciones colaboradoras. El objetivo principal } \\
\text { parte de la adquisición de un conocimiento sobre las ciudades para hacer un } \\
\text { mejor aprovechamiento de las mismas y de las oportunidades que otorgan los } \\
\text { flujos migratorios. }\end{array}$ \\
\hline $\begin{array}{l}\text { Portal para entidades } \\
\text { locales sobre } \\
\text { integración de } \\
\text { inmigrantes }\end{array}$ & $\begin{array}{l}\text { Portal web que ofrece recursos en materia de integración de inmigrantes, como } \\
\text { son: proyectos y experiencias exitosas, documentos, entrevistas y reportajes, } \\
\text { principales entidades que trabajan en el ámbito de la integración, etc. }\end{array}$ \\
\hline \multicolumn{2}{|c|}{ Gestión de la diversidad cultural en las empresas y organizaciones: } \\
\hline $\begin{array}{l}\text { Gestión de la diversidad } \\
\text { cultural en entornos } \\
\text { profesionales. Cuaderno } \\
\text { para la formación. } \\
\text { Cepaim }\end{array}$ & $\begin{array}{l}\text { Es un documento de formación para promover el asesoramiento a empresas y } \\
\text { organizaciones en la aplicación de herramientas de gestión de la diversidad. } \\
\text { Apuesta por la inclusión de las diferencias a nivel interno y externo y como eje } \\
\text { generador de valor en empresas y organizaciones. Está estructurado en cuatro } \\
\text { unidades para abordar la gestión de la diversidad. }\end{array}$ \\
\hline $\begin{array}{l}\text { Manual de Formación } \\
\text { en Gestión de la } \\
\text { Diversidad. Comisión } \\
\text { Europea}^{6} \text {. }\end{array}$ & $\begin{array}{l}\text { Es un manual elaborado por la Comisión Europea, dirigido a las empresas, } \\
\text { directivos y empleados sindicales. Ofrece información sobre gestión de la } \\
\text { diversidad y ejemplos de aplicación, incluida una herramienta de autoevaluación. }\end{array}$ \\
\hline $\begin{array}{l}\text { Un lugar seguro, } \\
\text { llamado prevención }^{7}\end{array}$ & $\begin{array}{l}\text { Es una guía para practicar la igualdad en el trato en el ámbito laboral del sector } \\
\text { agrario y en particular en materia de gestión de la diversidad y de prevención de } \\
\text { riesgos laborales. }\end{array}$ \\
\hline $\begin{array}{l}\text { Libro Blanco de la } \\
\text { Diversidad: una apuesta } \\
\text { por la gestión eficiente } \\
\text { de las personas en las } \\
\text { organizaciones. }^{8}\end{array}$ & $\begin{array}{l}\text { En el texto se presentan las aportaciones de expertos que han logrado tratar la } \\
\text { diversidad como una oportunidad de mejorar la organización. Asimismo, presenta } \\
\text { ejemplos de gestión de la diversidad cultural en tres grandes empresas. }\end{array}$ \\
\hline
\end{tabular}

2 http://www.ceimigra.net/observatorio/images/stories/luis_pdf/Manual_de_intervencin_comunitaria_en_barrios_2.pdf

3 http://www.opencities.eu/download/comprender_opencities.pdf

4 http://www.juntadeandalucia.es/justiciaeinterior/opam/es/node/816

5 http://cepaim.org/wp-content/uploads/2014/05/Gesti\%C3\%B3n-de-la-diversidad-Modulo-01-CEPAIMweb.pdf

6 http://www.idm-diversity.org/files/EU0708-TrainingManual-es.pdf

7 http://chil.me/download-file/104758-178623

8 http://www.fundaciondiversidad.org/libro_blanco_diversidad/LIBRO_BLANCO_DIVERSIDAD.pdf 


\section{CUADRO 4: Recursos para la gestión de la diversidad cultural en diferentes ámbitos de actuación (Continuación)}

\begin{tabular}{|l|l|}
\hline \multicolumn{2}{|l|}{ Gestión de la diversidad cultural en educación: } \\
\hline $\begin{array}{l}\text { Guía de Recursos de } \\
\text { Educación para el } \\
\text { desarrollo }\end{array}$ & $\begin{array}{l}\text { Es una guía formato Web que permite consultar materiales y experiencias de } \\
\text { Educación para el Desarrollo que están desarrollando las ONGD pertenecientes a } \\
\text { la Coordinadora de ONG para el Desarrollo-España. Ofrece metodologías } \\
\text { aplicadas con el objeto de que puedan llevarse a la práctica. }\end{array}$ \\
\hline $\begin{array}{l}\text { Red de Educación } \\
\text { Intercultural10 }\end{array}$ & $\begin{array}{l}\text { Es una página web que aporta diferentes recursos y asignaturas con contenido } \\
\text { especializado para formar al profesorado sobre diversidad cultural e incluye } \\
\text { estrategias para promover la interculturalidad en las aulas. }\end{array}$ \\
\hline Cuaderno Intercultural ${ }^{11}$ & $\begin{array}{l}\text { Se trata de una página web que incluye diferentes recursos para la } \\
\text { interculturalidad y la educación intercultural. Ofrece pestañas con diferentes } \\
\text { categorías, como: orientación docente, materiales didácticos, lectura y escritura, } \\
\text { dinámicas y juegos, herramientas, etc. }\end{array}$ \\
\hline Gestión de la diversidad cultural en salud: \\
\hline $\begin{array}{l}\text { Gestión de la diversidad } \\
\text { cultural en el ámbito } \\
\text { sanitario }\end{array}$ & $\begin{array}{l}\text { Programa formativo online dirigido a profesionales y estudiantes del ámbito de } \\
\text { la salud para el desarrollo de competencias de gestión de la diversidad }\end{array}$ \\
\hline $\begin{array}{l}\text { Guía de gestión de la } \\
\text { diversidad religiosa en } \\
\text { los centros hospitalarios }\end{array}$ & $\begin{array}{l}\text { Guía donde se proponen actuaciones para el ejercicio de la libertad religiosa } \\
\text { en los centros hospitalarios. Presenta el marco jurídico que regula la libertad } \\
\text { religiosa; aporta recomendaciones para su gestión; clarifica aspectos relacionados } \\
\text { con la dieta, la donación y trasplante de órganos y las posturas de las diferentes } \\
\text { confesiones. }\end{array}$ \\
\hline $\begin{array}{l}\text { Mediación intercultural } \\
\text { en el ámbito de la salud }\end{array}$ & $\begin{array}{l}\text { Guía formativa que contextualiza la mediación intercultural en el ámbito de la } \\
\text { salud y ofrece herramientas y conocimientos para la intervención dando ejemplos } \\
\text { concretos sobre diferentes grupos étnicos }\end{array}$ \\
\hline
\end{tabular}

Fuente: Elaboración propia.

\section{Ejemplos de proyectos y actividades}

La gestión de la diversidad "viene a reflejar la asunción implícita de lo diverso como determinante de lo organizativo y la necesidad de impulsar una serie de prácticas organizativas y de gestión que sean capaces de aprovechar el potencial de la diversidad en beneficio de todos" (Essomba 2008:12).

\footnotetext{
9 http://guiarecursos-epd.coordinadoraongd.org/

$10 \mathrm{http} / / / w w w . f u n d a c i o n f i d e . o r g / r e d /$ categoria/paginas_web.html

$11 \mathrm{http}: / /$ www.cuadernointercultural.com/

$12 \mathrm{http} / / / w w w$.asociacionmatiz.org/index.php/auladeformacion/133-diversidad-cultural-sanitario

13 http://www.mscbs.gob.es/profesionales/saludPublica/prevPromocion/promocion/desigualdadSalud/docs/Guia_gestion_diversidad_religiosa.pdf

${ }^{14}$ https://multimedia.caixabank.es/lacaixa/ondemand/obrasocial/pdf/inmigracion/Mediacio_intercultural_es.pdf
} 
Muchos de los ejemplos que nos encontramos en cuanto a gestión de la diversidad cultural se aplica en las empresas y organizaciones, aunque también se implementa a través de la educación y en los territorios (ciudades, barrios, etc.).

\subsection{Gestión de la diversidad en el territorio:}

Cuando hablamos del territorio, nos referimos tanto a ciudades y pueblos como a barrios. La gestión de la diversidad está vinculada al mundo local y a su capacidad de organización, grandes ciudades como New York, Bombay o Barcelona suelen ser referentes en la diversidad cultural. En este texto nos ocupamos de otros ejemplos que se desarrollan en contextos locales de tamaño medio.

EI Proyecto de Intervención Comunitaria Intercultural (ICI) $)^{15}$ actualmente se desarrolla en 40 territorios españoles gestionados por diferentes entidades sociales. El proyecto implementa una misma práctica comunitaria basada en la participación de la ciudadanía, con una estrategia y metodología comunes, y bajo una coordinación universitaria. Hasta la fecha ha contribuido al reto que supone gestionar adecuadamente la diversidad cultural a nivel local, a través de una gestión positiva de la diversidad cultural, étnica, lingüística y religiosa, de forma pacífica, preventiva y participativa.

En la ciudad de Madrid, se gestiona un programa de dinamización de los espacios públicos diseñado para formar comunidades interculturales, dentro del Plan de Convivencia de la ciudad. Uno de sus objetivos es promover el conocimiento intercultural entre nuevos vecinos permitiéndoles celebrar sus actos culturales tradicionales, y crear formas de ampliar la convivencia en los espacios públicos apoyando las reuniones interculturales en escuelas, parques, campos de deportes, y demás instituciones de la ciudad. Cada año se celebran cientos de actividades con decenas de miles de participantes que incluyen desde exposiciones sobre el racismo, celebraciones de una etnia concreta, fiestas religiosas, ligas deportivas o talleres intergeneracionales.

El Consejo y la Comisión Europea promueven la Red Intercultural Cities que tiene como objetivo hacer políticas de integración a nivel local desde el enfoque intercultural. La diversidad cultural es considerada como una oportunidad y las estrategias de integración se implementan de un modo transversal en diferentes áreas y niveles de gobierno. La Red pone a disposición de las ciudades una serie de herramientas que faciliten esta labor y evalúa los progresos realizados en los municipios integrantes. Entre estas herramientas destacan una recopilación de buenas prácticas, estudios e investigaciones y una guía paso a paso para la aplicación práctica del modelo de integración intercultural.

15 https://obrasociallacaixa.org/es/pobreza-accion-social/interculturalidad-y-cohesion-social/proyectode-intervencion-comunitaria-intercultural/que-hacemos 
El ayuntamiento de Barcelona puso en marcha en el año 2010, una estrategia para contrarrestar los rumores negativos sobre la diversidad cultural que combinaba diferentes acciones como la formación y sensibilización ciudadana o la creación de una red de "agentes antirrumor"16. Esta estrategia tuvo un impacto muy positivo a nivel local y empezó a implementarse en otros lugares tanto en España como en Europa siendo una estrategia de referencia por su enfoque y metodología innovadores. Aunque se desarrolla con elementos comunes, permite incluir actividades diversas y creativas adaptadas a la idiosincrasia de cada lugar.

\subsection{Gestión de la diversidad cultural en las empresas y organizaciones}

Actualmente es uno de los grandes campos donde se está implementando la gestión de la diversidad cultural, la cual se integra como parte de la cultura organizacional.

Un ejemplo de esto lo podemos ver en BSH Electrodomésticos donde potencian la experiencia internacional y el intercambio cultural de sus empleados a través de un programa de expatriados para sus colaboradores.

Los hoteles $\mathrm{NH}$ han firmado un Plan de Igualdad NH que establece 100 acciones que garantizan y promueven la igualdad de oportunidades. Se realiza un seguimiento de las candidaturas e incorporaciones con análisis estadísticos de género, edad y cultura. Cuentan además con un código de integración, respeto, no discriminación e igualdad de oportunidades que les ha permitido, más que registrar barreras o incidentes, detectar infinitas oportunidades y dotarse de flexibilidad.

El grupo Mahou-San Miguel cuenta con un manual del entrevistador donde se incorporan aspectos como diversidad o igualdad, estándar para todos los procesos. Cada persona que se incorpora al grupo tiene un proceso de adaptación, un itinerario diseñado a medida donde se recibe formación formal sobre igualdad, prevención de riesgos, sobre el aspecto concreto que va a desarrollar.

En Starbucks la gestión de la diversidad cultural se ha integrado en el núcleo del negocio. Cuentan con un equipo de gestión de la diversidad (Diversity and Inclusion Team) que tiene entre otros objetivos construir una plantilla diversa y crear una cultura inclusiva abierta a minorías y a las características culturales de las comunidades donde tiene presencia.

Otro ejemplo destacado lo encontramos en Air Products donde desarrollan el programa "Valorando la diversidad". En programa incluye la formación en sensibiliza-

16 http://www.antirumores.com/proyecto.html 
ción, equipos de liderazgo en la diversidad en cada región y la creación de asociaciones de empleados tales como "Empleados diversos étnicamente" o "Todos Americanos y Asiáticos".

\subsection{Gestión de la diversidad cultural en la Educación}

El Centro de Formación Padre Piquer de Madrid ha desarrollado transversalmente la gestión de la diversidad a través de un Plan para la convivencia ${ }^{17}$. Entre los proyectos que han implementado están las Aulas Cooperativas multitareas donde hay al menos 3 profesores simultáneos en el aula trabajando dos diferentes ámbitos: sociolingüístico y científico-tecnológico.

La Biblioteca Humana es una de las actividades de educación no formal implementadas en el municipio de Valongo, Portugal. Está dirigida a adolescentes de 14 a 18 años de edad y se realiza en colaboración con escuelas locales. Siguiendo un modelo lúdico basado en una biblioteca escolar, la clase visita la biblioteca y reserva un libro por un período determinado. Los libros de la Biblioteca Humana son voluntarios que representan a grupos culturales diversos y que cuentan historias de la vida real y que entabla diálogo con los lectores adolescentes. Estos se organizan en pequeños grupos para responder a estas historias y hablar sobre sus propios prejuicios y estereotipos. La meta es deconstruir estereotipos bajo el lema: «No juzgues un libro por su portada».

La ONG canadiense Equitas en colaboración con la ciudad de Montreal desarrolla un programa llamado ¡Juego justo! que tiene entre sus objetivos prevenir el racismo y la discriminación y preparar a los niños/as a convivir en una sociedad multicultural. Se implementa a través de programas de educación no formal y está destinada a niños y jóvenes de 12 a 16 años. El programa ha desarrollado más de 60 juegos y actividades para promover los valores centrales de los derechos humanos como la cooperación, el respeto, la justicia, la inclusión, el respeto a la diversidad, la responsabilidad y la aceptación. El éxito del programa radica en que después de los juegos, se les da voz a los niños y niñas para compartir sus sensaciones a través de debates sobre los objetivos de los mismos.

\subsection{Otras Actividades para la gestión de la diversidad cultural}

Además de los ejemplos que se han expuesto se presentan a continuación algunas actividades fáciles de realizar para comenzar a trabajar la diversidad cultural con grupos en el ámbito local.

17 http://www.padrepiquer.com/pdf/AulaCooperativaMultitarea.pdf 
La primera consiste en realizar un "Termómetro de la Convivencia". A partir de la representación de un termómetro se mide el grado de convivencia de un lugar concreto. Primero, se solicita a las personas participantes que en pequeños grupos valoren en una escala del 1 al 10 la convivencia del lugar. Después se debate por qué se ha elegido esa puntuación en vez de una menor. El resultado favorece ver los puntos positivos que hay en el lugar en cuanto a la convivencia, a partir de los cuales se puede empezar a trabajar.

La segunda se trata de promover el encuentro y conocimiento del otro, a través de la organización de comidas o cenas con "La familia de al lado". Esta actividad se ha organizado exitosamente en muchos lugares de Europa. Consiste en organizar comidas o cenas donde se junten una familia local y otra de origen extranjero donde cocinen y compartan la comida juntas. A través de estos encuentros se pone en comunicación a personas que, a pesar de vivir cerca, no han tenido la oportunidad de relacionarse en un contexto de mayor intimidad, y trabajar así las relaciones entre personas culturalmente diversas.

\section{Consideraciones finales}

Las sociedades del siglo XXI se caracterizan por ser plurales, heterogéneas y diversas, como nunca antes en la historia ha sido. La globalización y las nuevas tecnologías favorecen nuevas formas de interacción y nuevos flujos de movimientos de población. La gestión de la diversidad cultural parece haber superado los paradigmas etnocentristas y de relativismo cultural, en favor de enfoques interculturalistas. Es una cuestión de política, en todos los ámbitos (social, sanitario, educativo, empresarial). Y existen numerosos ejemplos que muestran, incluso en términos económicos, la suma positiva de una política de gestión de la diversidad cultural. Asimismo, la ONU ha señalado la riqueza que la diversidad cultural supone.

En este contexto, resulta relevante el papel del Trabajo Social como disciplina de referencia en la gestión de problemas sociales y garantía de Derechos Humanos, tal como destaca Nada al Nashif, subdirectora general de Ciencias Sociales y Humanas de la Unesco (2018) quien reconoce la estrecha relación entre ambos conceptos.

Las diferentes experiencias a las que nos enfrentamos desde elTrabajo Social para la gestión de la diversidad cultural implican repensar las metodologías de intervención y los modos de dirigirnos a los colectivos y personas con los que trabajamos. En este sentido, la intervención comunitaria es una herramienta que facilita estas acciones, ya que garantiza que va a existir una relación, desde el respeto de las diferencias, poniendo énfasis en lo común y promoviendo una interacción positiva. La clave para lograr lo anterior es la interculturalidad, en el sentido de crear contextos en los que se hable desde el "nosotros", sin diferenciar procedencias, etnias, culturas y otros aspectos que generan exclusión social. Por medio de un trabajo intercul- 
tural se logra que los colectivos a los que nos dirigimos descubran lo común y obvien las diferencias que pudieran existir. Por ello, trabajar desde la comunidad implica buscar espacios o momentos para crear situaciones de encuentro y dejar de distinguir entre el nosotros y el ellos, teniendo en cuenta una perspectiva integral de la sociedad y del individuo favoreciendo su empoderamiento y los procesos de participación.

Para dar respuesta a todo lo anterior, desde nuestra profesión tenemos que formarnos en competencias culturales y así favorecer estos intercambios y este hacer común. Del mismo modo, podemos incorporar elementos que faciliten esta labor como, por ejemplo, combinar el arte y la creatividad en estos espacios de participación ciudadana. Lo importante de lo anterior es el proceso en sí mismo, más allá de los resultados que éste pueda originar. Estos procesos tienen que considerar a las personas como protagonistas, y por lo tanto como sujetos y no objetos de la acción. Durante el proceso tienen lugar los encuentros y las vivencias, además de los momentos en los que se comparte y se gestiona la diversidad cultural la interacción positiva y el conocimiento de personas de distintas culturas ayudando a superar prejuicios y la fragmentación social.

\section{Referencias Bibliográficas}

Al-Nashif, N. 2018. "Human Right and Social Work", Trabajo Social Global, Vol 8, n extraordinario:9-18. Granada: Universidad de Granada. DOI https://dx.doi.org/10.30827/tsggsw.v8i0.7463

De las Heras, P. y Cortajarena, E.. 1985. Introducción al Bienestar Social, Madrid: Siglo XXI.

Escarbajal Frutos, A. 2012. Interculturalidad, mediación y trabajo colaborativo. Madrid: Narcea.

Essomba, M.A. 2013. "Las competencias básicas en interculturalidad". Jornada de Formación para profesionales y otros actores que intervienen en contextos de diversidad cultural, celebrada en Marzo de 2013 en Logroño (La Rioja), organizada por la Obra Social La Caixa.

Essomba, Miguel Angel. 2008. Diez ideas clave. La gestión de la diversidad cultural en la escuela. Barcelona: Graó.

Festinger, L. 1957. A Theory of Cognitive Dissonance. Stanford: Stanford University Press.

Giménez, Carlos. 2003. "Pluralismo, multiculturalismo e interculturalidad". Educación y Futuro n 8, Barcelona: Editorial CES Don Bosco, Edebé. Disponible en

http://www.redeseducacion.net/articulos/Materiales/Interculturalidad/c. \%20gimenez\%20pluralismo\%20multiculturalismo\%20interculturalidad.pdf

Giménez, C., Álamo, J.M. y Pérez del Olmo, F. 2015. "Convivencia y cohesión social", en Juntos por la Convivencia. Claves del Proyecto de Intervención Comunitaria Intercultural, $n^{\circ}$ 1. Barcelona: Obra Social "La Caixa".

https://obrasociallacaixa.org/documents/10280/583314/juntos_por_la_convivencia_convivencia_y_cohesion_social_es.pdf/8bd5af66-b40c-4b13-9ea6-f15cb783412f 
Hernández Correa, P.J. (2007). “Educación y desarrollo comunitario: dialongando con Marco Marchioni" Cuestiones Pedagógicas, 18: 285-300. Sevilla: Universidad de Sevilla.

Kisnerman, N. (1984) Teoría y práctica del trabajo social. Los recursos. Vol. 4. Buenos Aires: Humanitas

Marchioni, M. (1989). Planificación social y organización de la comunidad.

Alternativas avanzadas a la crisis. Madrid: Popular.

Martín, L. (2003) "Escuela y diversidad lingüística y cultural". En ¿Asimilar o integrar? Dilemas ante el multilingüismo en las aulas. Madrid: Ministerio de Educación, Cultura y Deporte: 19-68.

http://www.equintanilla.com/Documentos/escuela\%20y\%20diversidad\%20linguistica.pdf

Martínez Aranda. A. 2015. "Los espacios públicos como lugares de recreación identitaria: elementos de conflicto en contextos locales de inmigración." En Giménez Romero, C. y Gómez Crespo, P. 2015. Análisis, prevención y transformación de conflictos en contextos de migración." Madrid: UAM Ediciones.

Moix, M. 2004. El Trabajo Social y los Servicios Sociales. Su concepto. En Cuadernos de Trabajo Social, Vol. 17:131-141: Madrid: Universidad Complutense de Madrid.

Moreno Moreno, J. 2006. "La mediación en el ámbito de la inmigración y convivencia intercultural". Zaragoza: Universidad de Zaragoza. Acciones e investigaciones sociales, $N^{\circ}$ Extra 1.

Ramasco Gutiérrez, M. y Garabato González, S. 2015. “Estrategias de intervención en salud con perspectiva intercultural." En Giménez Romero, Carlos, Gómez Crespo, Paloma. (coord..) Análisis, prevención y transformación de conflictos en contextos de migración." Madrid: UAM Ediciones.

Rinken, S. 2011. "La gestión de la diversidad cultural en Andalucía: buenas prácticas y retos de futuro". En Sánchez, Elena. y Pinyol, Gemma. (coord.). Políticas públicas y modelos de ciudadanía, págs. 139-151. Barcelona: Fundación CIDOB.

Vázquez, O. 2002. Trabajo Social y Competencia Intercultural, en Portularia, $n^{\circ} 2: 125-$ 138, Universidad de Huelva, disponible en http://rabida.uhu.es/dspace/bitstream/handle/10272/140/b12151907.pdf;jsessionid=C224CB2CB970ABEA580787960AF8A860?seq uence $=1$

Plog, F. y Bates, D. 1980. Cultural Anthropology. New York: Alfred Knoff.

Portes, Alejandro, Vickstrom, Erik. 2012. "Diversidad, capital social y cohesión". Revista Española de Sociología, n 17: 83-107. Madrid. Federación Española de Sociología

Löwy Gundel, Claudio. 2006. El concepto de cultura vinculado a las necesidades. Programa Permanente de Capacitación a Escala Humana. Buenos Aires: Fundación Unida. www.unida.org.ar/.../Modulo\%20l\%20Cultura\%20vinculada\%20a\%20necesidades.do... 


\section{comunitania}

REVISTA INTERNACIONAL DE TRABAJO SOCIAL Y CIENCIAS SOCIALES

ARTICULOSS/ARTILLES

Anti-oppressive education: Messages from Paulo Freire / Educación anti-opresiva: mensages de Paulo Freire

Sofia Dedotsi y Efrosyni-Alkisti Paraskevopoulou-Kollia

Págs 9-20

Programa disfruta de la experiencia, voluntariado de mayores y envejecimiento activo en el medio rural

-Andorra (Teruel) / Enjoy the experience: a program about senior

volunteering and active aging in rural environment -Andorra (Teruel)

Margarita Alloza, Meritxel Garcés, Ana Lázaro, Agustín Quílez, Teresa Sánchez y María José Muñoz

Págs 21-35

Actitudes hacia la diversidad. La discriminación y el estigma en estudiantes de trabajo social mexicanos /

Attitudes towards diversity. Discrimination and stigma in students of mexican social work

Luis M. Rodríguez Otero y Lydia del Carmen Ávila Zárate

Págs 37-63

Gestión de la Diversidad Cultural: recursos y herramientas del Trabajo Social / Management of Cultural

Diversity: Resources and Tools of Social Work

Esther Raya-Diez, Maria Ezquerro Sáenzn y Cecilia Serrano-Martinez

Págs 65-84

Anti-Roma prejudice in Greece: A challenge for social work / Prejuicio contra los romaníes en Grecia:

Un desafío para el trabajo social

Charis Asimopoulos, Sofia Martinaki, Despoina Kompoti, Eleni Kontogianni y Georgia Gouga

Págs 85-100

\section{RESENATAS/REVIIEWS}

Pastor Seller, E., Cabello Garza, M.L. (eds.) 2018. Retos y desafíos del trabajo social en el siglo XXI:

Una perspectiva internacional comparada México-España / Social Work challenges in the XXI Century: An international comparation between México-Spain. Madrid: Dykinson

(por Rafael Acebes Valentín)

López Peláez, A., Gómez Ciriano, E.J. 2019. Austerity, Social Work and Welfare Policies: a Global Perspective / Austeridad, Trabajo Social y Políticas de Bienestar: Una perspectiva global. Pamplona:Thomson-Reuters Aranzadi

(por Emilio Díaz de Mera)

Págs 105-107 